East African Medical Journal Vol. 85 No. 6 June 2008

SUCCESSFUL PREGNANCY OUTCOME FOLLOWING LAPAROSCOPIC MYOMECTOMY: CASE REPORT

R.B. Parkar, MBBS, MMed (Obst/Gyn), FAGE, Dip. Lap. Surg., Consultant Obstetrician and Gynaecologist, P.O. Box 520-00606, Nairobi, Kenya, S. Wanyonyi, MBChB, Post Graduate Resident in Obstetrics and Gynaecology, The Aga Khan University Hospital, Nairobi, P.O. Box 30270-00100, Nairobi, Kenya, W.J. Kamau, MBChB, MMed, Consultant Obstetrician and Gynaecologist, P.O. Box 19794-00200, Nairobi, Kenya and D. Otieno, MBChB, MMed, Consultant Anaesthetist, P.O. Box 19990-00202, Nairobi, Kenya

Request for reprints to: Dr. R.B. Parkar, P.O. Box 520-00606, Nairobi, Kenya

\title{
SUCCESSFUL PREGNANCY OUTCOME FOLLOWING LAPAROSCOPIC MYOMECTOMY: CASE REPORT
}

\author{
R.B. PARKAR, S. WANYONYI, W.J. KAMAU and D. OTIENO
}

\begin{abstract}
SUMMARY
Laparoscopic myomectomy is now widely used as an alternative to laparotomy in the management of symptomatic uterine fibroids. The advantages of this minimal access approach outweigh those of the open techniques. The pregnancy outcomes between the two methods have been studied and are comparable, but there still exists a lot of scepticism locally concerning this. It is against this background that we present a 31 year old nulliparous lady who had a two year history of primary infertility secondary to multiple uterine fibroids, the largest being fundal and measuring $6.6 \mathrm{~cm}$. She underwent a successful laparoscopic myomectomy in November, 2006 and conceived spontaneously in February 2007. Her antenatal follow up was uneventful. She delivered a live male, 2,650 grams by Caesarean section in October, 2007 and had an unremarkable peuperium. With the availability of proper equipment, instruments, and adequately developing skills, laparoscopic myomectomy is feasible locally and with proper patient selection could result in favourable outcome as it is elsewhere.
\end{abstract}

\section{INTRODUCTION}

Traditionally abdominal myomectomy has been the treatment of choice for uterine myomas especially in women desiring fertility. Besides abnormal bleeding, myomas could distort the uterine cavity or obstruct the tubal ostia resulting in infertility. Other mechanisms that could result in infertility include alternations in the endometrium affecting embryo implantation and growth (1).

Myomectomy for symptomatic uterine fibroids could either be done laparoscopically or by laparotomy. With the availability of instruments and adequate sustained training especially in intracorporeal suturing techniques, laparoscopic myomectomy is increasingly gaining favour globally (1). Despite its limitations with regards to the numbers and location of the fibroids, laparoscopic myomectomy has several advantages when compared to the open approach (2).

These include reduced blood loss at surgery, less post operative pain, shorter hospitalisation, faster recovery, less febrile and fewer surgical complications (2). Its impact on the obstetric outcome in mothers desiring fertility has been a matter of discussion. There has been uncertainty as to the integrity and strength of the myomectomy scar. To address these genuine concerns a carefully designed prospective study undertaken by Seracchioli et al (3) concluded that laparoscopic myomectomy was comparable to laparotomic myomectomy in terms of restoring fertility and pregnancy outcome and has well known advantages. Several retrospective studies and case series have had similar findings (4-6). In spite of these 
advances, the technique has not gained acceptance locally. There could be several factors contributing to this slow progression, but the most significant is the lack of the expertise in intra-corporeal suturing techniques, as laparoscopic myomectomy is an advanced laparoscopic procedure.

With only $6.3 \%$ of gynaecologists in Nairobi being able to perform level III minimal access surgical procedures, this could be an anticipated drawback (7). Local experiences in gynaecological laparoscopy have been published with laparoscopic myomectomy being reported among other procedures including total laparoscopic hysterectomy and laparoscopic subtotal hysterectomy and ovarian surgery $(8,9)$. With numerous laparoscopic procedures being performed, it is worthwhile reporting on the outcomes to allay any unfounded fears. It is against this background that we present a case of a successful pregnancy outcome following laparoscopic myomectomy at the Aga Khan University Hospital in Nairobi. The case is presented, not because it is peculiar to the world of reproductive health, but to the best of our knowledge it is the first case managed and reported locally. We are cognisant of numerous other successes reported elsewhere $(10,11)$. We hope that laparoscopic myomectomy would gain acceptance locally, so that women could enjoy the vast advantages offered by minimal access surgery.

\section{CASE REPORT}

A 31 year old nulliparous lady presented with a two year history of lower abdominal pains, heavy regular menstrual flow, non responsive to conventional therapy and failure to conceive after two years of marriage. The menstrual flow was heavy with clots and she changed more than seven sanitary pads a day during her cycles. She had no history of intermenstrual or post coital bleeding, dysuria or vaginal discharge. There was no history of any contraceptive use. An adequate evaluation was undertaken, and included a normal hormone profile and semen analysis. A pelvic ultrasound reported a "solitary" subserous fibroid of $6.6 \mathrm{~cm}$ arising from the uterine fundus. In addition a hysterosalpingogram showed a patent right fallopian tube, occluded left fallopian tube, and a fundal filling defect.

On general examination there was no pallor, cyanosis, icterus or lymphadenopathy. She was of good build with no hirsutism or oedema and her breasts were not active. The cardiovascular, respiratory and central nervous systems were normal.

Abdominal examination revealed no scars or areas of tenderness. The liver and spleen were not palpable. The uterus was 16-18 weeks in size and grossly irregular, and mobile. There was no evidence of any intrabdominal free fluid. The bowel sounds were normal.

On vaginal examination, the external genitalia were normal, with healthy vaginal mucosa. The uterine size, mobility and texture were confirmed as above. There was neither adnexal nor pouch of Douglas tenderness or masses. The cervix was firm and appeared normal. Her haemoglobin was $10.9 \%$ and the urea, electrolytes and blood sugar were all normal. She was blood group A positive.

The available options of management were considered and discussed and the patient opted to undergo a diagnostic hysteroscopy and laparoscopic myomectomy with a possibility of laparotomy if this was not feasible.

In theatre under general anaesthesia, aseptic precautions were observed. At hysteroscopy and under fluid distension using a $30^{\circ}$ Bertocchi hysteroscope, both the tubal ostia were clearly visualised. There were no submucous fibroids or any endometrial polyps seen. The endometrial cavity was rather distorted. The panoramic view and the endocervical canal appeared normal. An endometrial biopsy was obtained for histological evaluation.

After repositioning the patient, a pneumoperitoneum was created using a verres needle and $\mathrm{CO}_{2}$. Due to the size of the fibroid a supraumbilical $15 \mathrm{~mm}$ port was created (Lee Huangs point: midway between the umbilicus and xiphoid process) to introduce the laparoscope. Two suprapubic access routes (one $10 \mathrm{~mm}$ and one $5 \mathrm{~mm}$ ) were inserted lateral to the inferior epigastric arteries and slightly higher than usual.

The abdominal cavity was inspected and revealed a normal appendix, liver and gall bladder. The ovaries and fallopian tubes including the fimbriae were normal and the pelvic cavity was devoid of any endometriotic lesions. The uterus was 16-18 weeks in size and irregular with three large subserous fibroids ranging from $4-6 \mathrm{cms}$ each over the fundus right lateral wall and posterior uterine wall. In addition there were nine seedling 
fibroids approximately $2 \mathrm{~cm}$ each scattered over the anterior uterine wall. A laparoscopic myomectomy was undertaken through three uterine incisions on the serosa overlying the myomas using a harmonic scalpel. The myomas were enucleated by countertraction on the uterus and detached from their respective beds. In the process the endometrial cavity was beached. The uterine wall was sutured in one layer using vycril 1 / 0 with intracorporeal knots. The seedling fibroids were also removed using bipolar coagulation and sharp dissection.

All the myomas were retrieved from the abdominal cavity using an electronic morcellator through a $15 \mathrm{~mm}$ port. A saline lavage ensured adequate haemostasis and a final check revealed no visceral injuries. The estimated blood loss was $100 \mathrm{mls}$. The entire surgery lasted 110 minutes.

The patient recovered well and was ambulant and taking orally 6 hours after surgery. She was discharged on the third post-operative day and scheduled for follow up. The haemoglobin on discharge was 10.0gms / dl (Figures 1-3).

The histology confirmed uterine leiomyomata, total weight of the morcellated specimen being 160 grams.

She presented two months later having conceived spontaneously, her last normal period being February, 2007. A first trimester ultrasound confirmed a viable intrauterine pregnancy and she was subsequently enrolled for antenatal follow-up which was unremarkable.

Figure 1

Uterus with fibroids before myomectomy

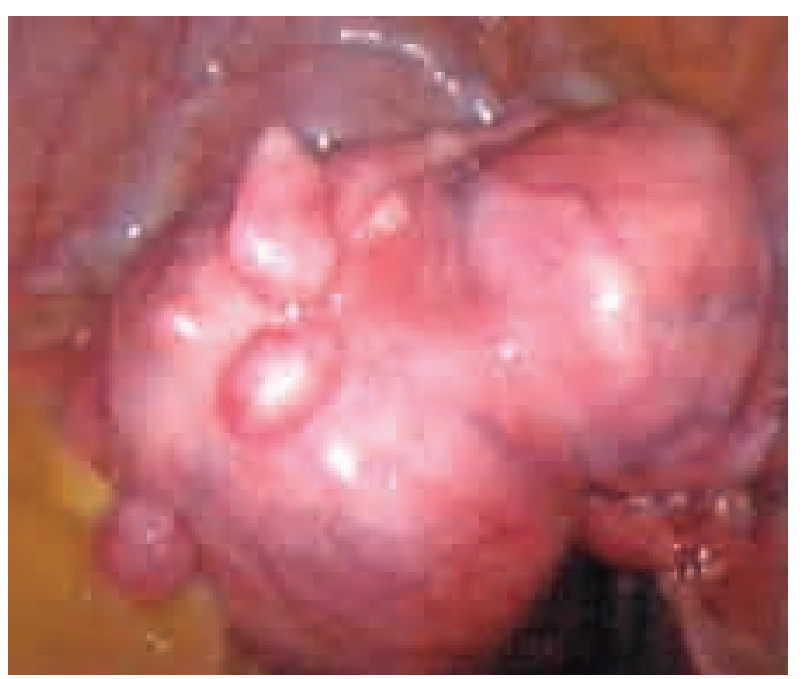

Figure 2

A posterior wall fibroid approximately $6 \mathrm{~cm}$ held with a myoma spiral

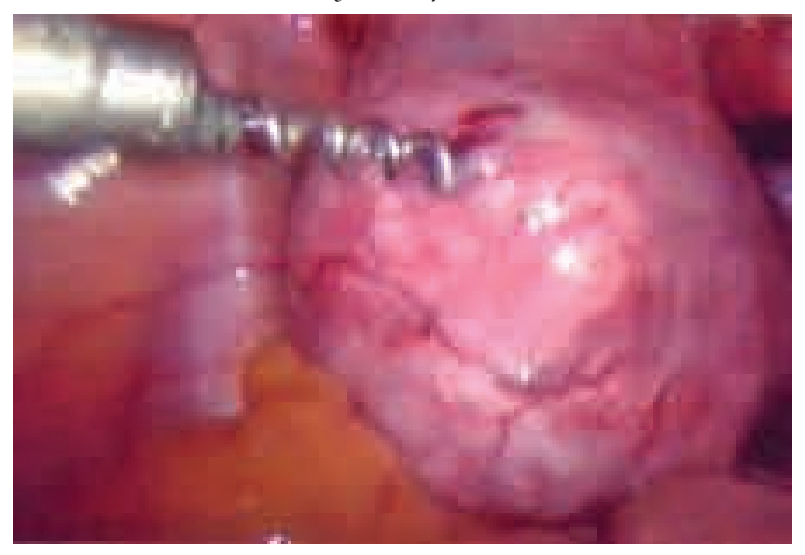

Figure 3

Appearance of the uterus after myomectomy

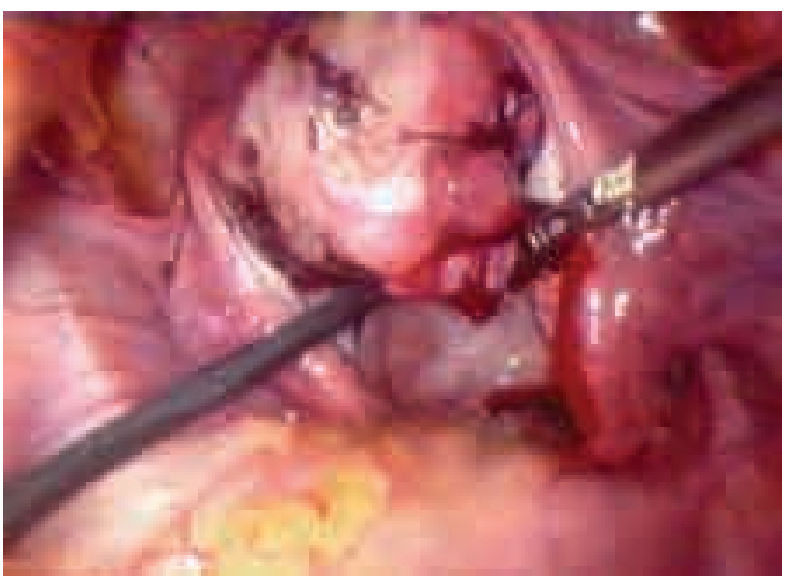

She was planned for an elective Caesarian section at 38 completed weeks since the uterine cavity had been breached. A live male baby was delivered by a routine lower uterine segment Caesarian section. The baby's apgar score was 10/1,10/5 and weighed 2650 grams. The placenta was non adherent and was delivered by controlled cord traction. No adhesions were encountered at surgery and the myomectomy sites were intact.

\section{DISCUSSION}

Successful pregnancy following laparoscopic myomectomy has been well documented $(10,11)$. Despite this, the use of minimal access surgery for the management of myomas is received with a lot of scepticism locally.

Although majority of fibroids may be asymptomatic in the presence of abnormal uterine 
bleeding not responsive to therapy, compression of adjacent structures like ureters, bowel, bladder, high level of suspicion of malignancy, growth after menopause, and infertility with distortion of the endometrium cavity or tubal occlusion, surgical intervention is advocated (12).

In the case presented, a 31 year old with menorrhagia, multiple large fibroids with a distorted uterine cavity was responsible for delayed fertility. Proper client selection is mandatory and a good preoperative ultrasound should be done to identify the number, location and size of the various fibroids. In our case there was a large discrepancy between the pelvic ultrasound and the laparoscopic findings.

Treatment options vary but counselling patients on the available options is advisable. When advising for laparoscopy the possibility of laparotomy should always be borne in mind. Laparoconversion should be seen as sign of wisdom rather than evidence of defeat (12).

Marret et al (13) in a retrospective multicentre study comparing myomectomy by laparoscopy and laparotomy, noted that laparoscopic myomectomy is a safe, reliable and reproducible surgical procedure, with less intraoperative blood loss and post operative haemoglobin drop. Although laparoscopic myomectomy required adequate and sustained skills to perform, it was observed that patients recovered faster and returned to work quicker. There was also a reduced risk of post operative adhesions formation, less febrile morbidity and it was recommended that laparoscopic myomectomy should always be the intended procedure (13).

Similarly Rosetti et al (14) in reviewing 332 patients who underwent laparoscopic myomectomy reported significant advantages. In the case presented, ambulation was immediate and discharge was on the third post operative day with full recovery by day 10. Modifications may be required e.g. placement of ports at convenient sites including the Palmer's point or having additional ports. The suturing technique could also vary. Favourable outcome with single layer suturing has been reported. The number of layers depends on the depth and extent of the cavity (16). Thermal damage and haematoma formation are the commonest causes of suboptimal healing and rupture during pregnancy (16-18), and these should be avoided.
This could be prevented by correct reapproximation with evenly spaced sutures to avoid haematomas and avoiding overaggressive control of bleeders using electrosurgical techniques as this could result in tissue ischaemia and necrosis. Cases of uterine rupture remote from term with either method of closure have been occasionally reported $(17,18)$.

The time interval before conception has not been determined, an arbitrary interval of six months is widely acceptable. The patient presented conceived two months after surgery and proceeded to term eventually. Planned elective Caesarian section is common in women who have undergone myomectomy especially in the presence of fundal fibroids and if the uterine cavity has been breeched. This is due to fear of uterine rupture.

Although Dubuisson et al (15) recommended that fibroids of less than $10 \mathrm{~cm}$ and not more than three in number were ideal for laparoscopic myomectomy, in our case there were three large fibroids and nine seedling fibroids. It was reported that laparoscopic myomectomy resulted in less pelvic and periadnexial adhesion formation thereby increasing the chances of fertility.

Well designed studies to determine the interval from surgery to conception and possibility of vaginal delivery for intramural fibroids would be recommended.

In conclusion, with the availability of proper instruments and training, laparoscopic myomectomy is feasible locally. Proper patient selection could result in favourable outcome as it is elsewhere. Women should however be presented with all the available options and guided into making proper choices. This case can't be conclusive on the favourable outcomes associated with laparoscopic myomectomy, more prospective studies should be undertaken as the approach becomes more available.

\section{ACKNOWLEDGEMENTS}

To the staff of the Aga Khan University Hospital theatre, our colleagues and the patient for allowing this publication. 


\section{REFERENCES}

1. Parker, W.H. Uterine myomas. Fertil. Steril. 2007; 88: 255-271.

2. Hurst, B.S., Mathews, M.L. and Marshburn, P.B. Laparoscopic myomectomy for symptomatic uterine myomas. Fertil. Sterl. 2005; 83: 1-23.

3. Seracchioli, R., Rossi, S., Govoni, F., et al. Fertility and obstetric outcome after laparoscopic myomectomy of large myomata: A randomised comparison with abdominal myomectomy. Human Repro. 2000; 15: 2663-2668.

4. Seracchioli, R., Manuzzi, L., Vianello, F., et al. Obstetric and delivery outcome of pregnancies achieved after laparoscopic myomectomy. Fertil. Steril. 2006; 86: 159-165.

5. Paul, P.G., Koshy, A.K. and Thomas, T. Pregnancy outcomes following laparoscopic myomectomy and single layer myometrial closure. Hum. Repro. 2006; 21: 3278-3281.

6. Dubuisson, J., Fauconnier, A., Deffarges, J., et al. Pregnancy outcome and deliveries following laparoscopic myomectomy. Hum. Reprod. 2000; 65: 996-1001.

7. Obura, T. Knowledge, attitude and practice of minimal access surgery (M.A.S.) amongst gynaecologists in Nairobi, Kenya, 2002. Unpublished MMed dissertation, University of Nairobi.

8. Parkar, R.B., Thagana, N.G. and Otieno, D. Laparoscopic assisted vaginal hysterectomy for benign uterine pathology: Is it time to change? East Afr. Med. J. 2004; 181: 261-265.

9. Parkar, R.B., Thagana, N.G., Baraza, R., et al. Experience with laparoscopic surgery at the Aga Khan University Hospital, Nairobi, East Afr. Med. J. 2003; 80: 44-50.
10. Landi, S., Fiaccavento, A., Zaccoletti, R., et al. Pregnancy outcomes and deliveries after laparoscopic myomectomy. J. Amer. Assoc. Gynecol. Laparosc. 2003; 10: $177-181$

11. Campo, S., Campo, V. and Gambadauro, P. Reproductive outcomes before and after laparoscopic or abdominal myomectomy for subserous or intramural myomas. Eur. J. Obstet. Gynecol. Reprod. Biol. 2003; 100: 215-219.

12. Wallach, E.E. and Vlahos, N.F. Uterine myomas: An overview of development, clinical features and management. Obstet. Gynecol. 2004; 104: 393-406.

13. Marret, H., Chevillot, M. and Giraudeau, B. A retrospective multicentrestudy comparing myomectomy by laparoscopy and laparotomy in current surgical practice. What are the best patient selection criteria? E. J. Obstet. Gynaecol. 2004; 117: 82-86.

14. Rosseti, A., Sizzi, O. and Soranna, L. Long term results of laparoscopic myomectomy: Recurrence rates in comparison with abdominal myomectomy. Hum. Reprod. 2001; 16: 770-774.

15. Dubuisson, J.B. and Chapron, C. Uterine fibroids: Place and modalities of laparoscopic treatment. Eur. J. Obstet. Gynaecol. Reprod. Biol. 1996; 65: 1-4.

16. Goldberg, J. and Pereira, L. Pregnancy outcomes following treatment for fibroids: Uterine fibroid embolisation versus laparoscopic myomectomy. Current Opin. Obstet. Gynecol. 2006; 19: 402-406.

17. Banas, T., Klimek, M., Fugiel, A., et al. Spontaneous uterine rupture at 35 weeks gestation, 3 years after laparoscopic myomectomy, without signs of fetal distress. J. Obstet. Gynecol. Res. 2005; 31: 527-530.

18. Jakiel, G., Sobstyl, M. and Swatowksi, D. Spontaneous uterine rupture during delivery in a patient who had previously undergone laparoscopic myomectomy. Gynecol. Endoscopy. 2002; 11: 315-317. 